\title{
Research on feedback control of the intelligent grid-connected photovoltaic systems based on micro-inverters
}

\author{
Fangmei Wang ${ }^{1, a}$, Rui Wang ${ }^{2, b}$, Reda Hassanien Emam Hassanien ${ }^{2, c}$, \\ Ming $\mathrm{Li}^{3, \mathrm{~d} *}$ Ziyang Zhang ${ }^{4, \mathrm{e}}$, . Jin Gao ${ }^{4, f}$
}

Solar Energy research Institute, Yunnan Normal University, Kunming, Yunnan 650500, China

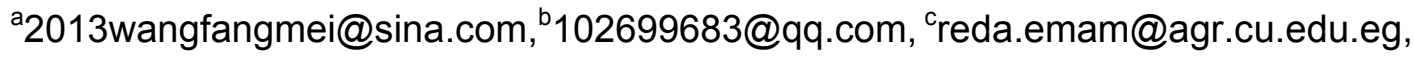

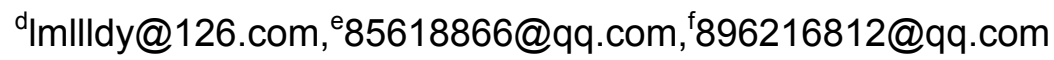

Corresponding Author: Ming Li

\begin{abstract}
Keywords: grid-connected PV system, micro-inverter, acquisition controller, intelligent feedback control, self-diagnose, autonomous maintenance
\end{abstract}

\begin{abstract}
Recently, the grid-connected photovoltaics power stations are extensively installed. Nevertheless, to save the maintenance time, improve the safety performance, solve the complexities of traditional overhaul methods and eliminate restrictions further research is needed. Therefore, in this paper, a method of self-diagnose control for photovoltaic system connected grid based on micro-inverter was proposed. The smartPV software used to monitor the parameters of each module and the whole system. Subsequently, we develop a new software to self-diagnose the specific location of the fault or shadow. The feasibility and rationality of the self-diagnose method to the system state were verified by a simulation model and experiment. Results showed that this method can provide a number of functions: such as, the sub-system grouping contrast, the system self-started, found the specific position of the battery plate, divide the degree of the shielding, and protect the system and the system self-closed. In conclusion, this method makes the maintenance of the photovoltaic connected grid more safe and efficient. Moreover, it can be applied to on-line maintenance and autonomous maintenance for the household and the large scale grid-connected photovoltaics systems.
\end{abstract}

\section{Introduction}

In recent years, the government has paid more attention to the environment. Therefore, solar energy has been developed intensively by technological improvements, government policies supportive, and the development of large-scale distributed photovoltaics(PV) system. Thereby, the household distributed grid-connected PV system is becoming more and more rapidly. Therefore, following the system running status monitoring and maintenance are important factors for the safe operation of photovoltaic power generation system. It is clear that most of the photovoltaic power plants nowadays are using the manual inspection and maintenance, and the electrical parameters of PV modules are detected by the block detection. However, the maintenance of PV modules installed

in high places and even up to hundreds of volts consume time and it is very dangerous. Therefore, the establishment of a feedback control system for on-line operation state detection is a great issue to be investigated[1].

The previous domestic and foreign studies have put forward some solutions. Wang et al.[1] analyzed the output characteristics of PV modules in the short circuit and abnormal aging

conditions. Furthermore, they established the fault type factor K, by comparing the difference between the $\mathrm{K}$ and the standard values. Meanwhile, the short-circuit and abnormal aging faults were estimated. Zhang et al. [2]proposed a double closed-loop control system based on quasi proportional resonant control for the current loop controller connect grid and the controller of the DC voltage outer loop based on the auto disturbance rejection control. In addition, Barghi Latran et al.[3] analyzed the power quality problems of a multi-level multi-functional inverter grid connected with low power consumption, low harmonic distortion and low electromagnetic interference 
output.Monfared et al.[4] pointed out that the current hysteresis control, voltage directional control and proportional resonance control should be paid more attention, they also discussed the technology of some practical aspects of the implementation. On another studies, the fault diagnosis method based on BP neural network was proposed to detect faults[5, 6]. It was reported that the voltage of three phase power network was unbalanced to be disturbed by the control method of drooping characteristic, and controlled by the frequency variation and harmonic distortion[7, 8]. Also, self-diagnostic method using distributed MPPT was proposed[9].

Nevertheless, the system can solve the problem of online operation state detection; there are some challenges in the implement. For Intelligent feedback control of the photovoltaic system to achieve a simple, safe and efficient purpose, a combination of micro-inverter technology and narrowband power line carrier communication technology (NB-PLC) of experimental platform was used.

This paper has developed a self-diagnose feedback control intelligent grid-connected PV systems based on micro-inverters and the platform of smart grid-connected PV systems. In order to decrease the maintenance time, improve the safety performance and eliminate restrictions, a new approach has been conducted for self-diagnosis online operating status type which can be determined corresponding to the specific location of the fault shadow, time and size.

Intelligent feedback control self-diagnosis has timers to ensure the feasibility of online testing and determination of the entire system running status. The system does not require the use of radiation measuring instrument, communication lines, and additional hardware. Moreover, reducing the installation costs and beautify the installation environment. The fault detection method is an accurate, intelligent control, warning statement popular, which can be applied to larger PV overhaul and autonomous maintenance.

\section{Construction of the whole system}

To achieve function of system online operational status detection, a power station of $10 \mathrm{KW}$ grid-connect PV system has been constructed, each group had four panels with dip, azimuth adjustable. Its structures based on micro-inverter grid-connected PV system, each panel was configured with a collection control channel, and each channel was composed of a collection module, a control module, a narrow band power line carrier communication module and a power supply module. Fig. 1 shows the entire PV system schematic diagram, when SI represents the inverter, MI represents micro-inverter, MIDACC represents micro-inverter data acquisition and control cabinet, NB-PLC represents narrow band power line carrier communication module. The physical map of grid- connected PV system based on the micro-inverter is shown in Figures 2 and 3.

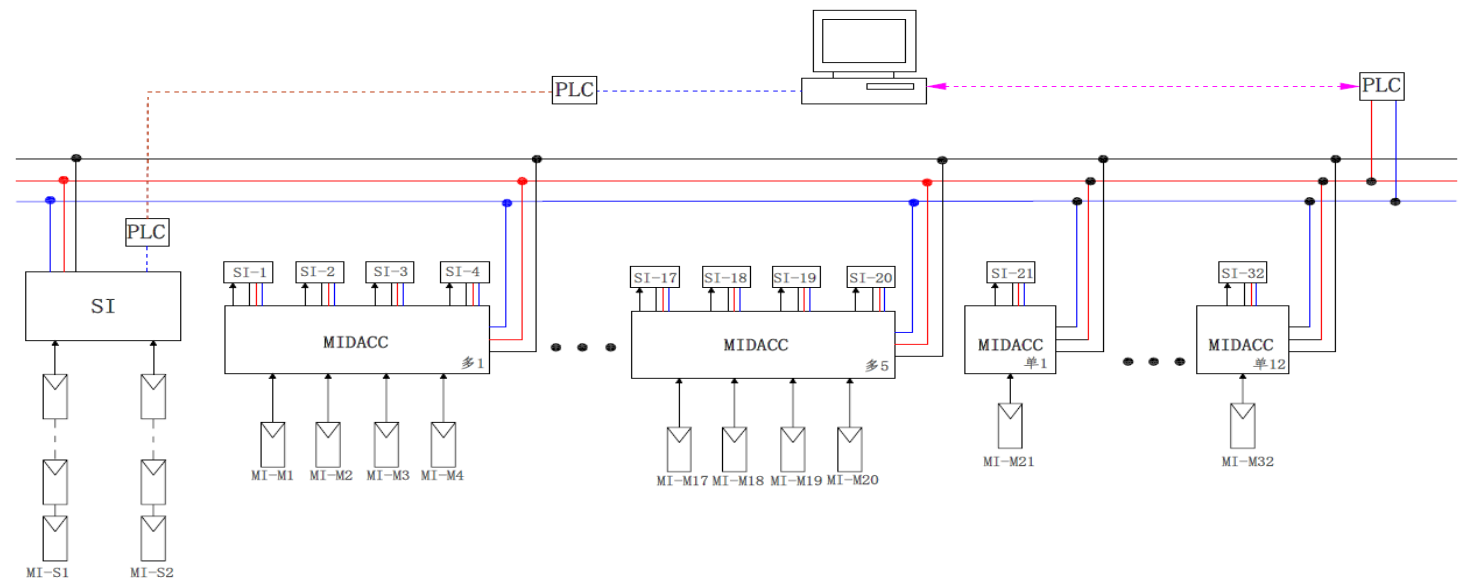

Fig 1.10KW PV system connect grid schematic 


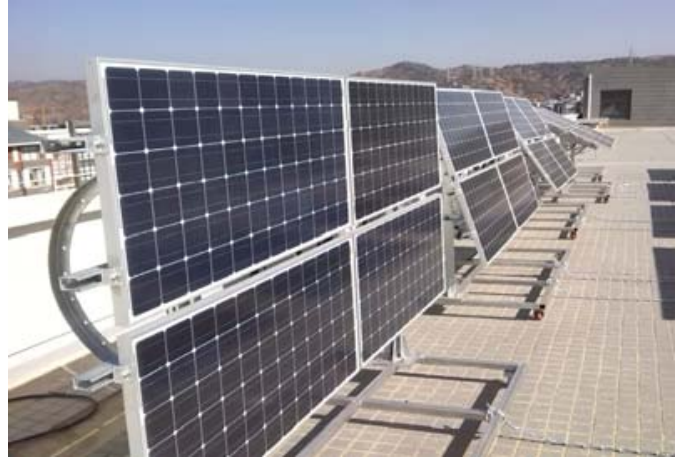

Fig. 2. System positive physical

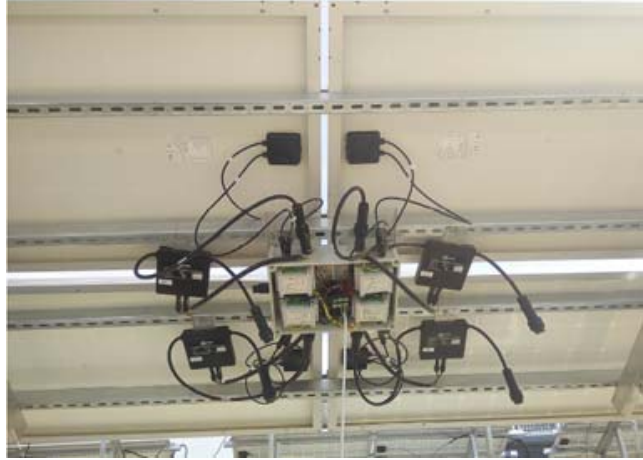

Fig. 3.System back diagram

PV system based on micro-inverter, each panel was equipped with a micro-inverter. To avoid insecurity, collector and controller were sealed in the box. Parameters of solar panels and micro-inverter system are shown in Table 1:

Table 1

\begin{tabular}{llllll}
\hline Category & Type & Parameters & Value & Parameters & Value \\
\hline Battery plate & \multirow{2}{*}{ SM572-190 } & Max-DC power & $190 \mathrm{~W}$ & Short circuit current & $5.52 \mathrm{~A}$ \\
& & Peak voltage & $36.5 \mathrm{~V}$ & Operating temperature & $-40^{\circ} \mathrm{C} \sim+85^{\circ} \mathrm{C}$ \\
& & Peak current & $5.21 \mathrm{~A}$ & scale & $1580 \times 808 \times 40$ \\
\multirow{2}{*}{ Micro-inverter } & \multirow{2}{*}{ YC250A } & Open-circuit voltage & $44.5 \mathrm{~V}$ & Cell Technology & Moni-Si \\
& & DC Current & $1-5.52 \mathrm{~A}(5.21 \mathrm{~A})$ & AC voltage & $210-240 \mathrm{~V}$ \\
& & DC voltage & $1-44.5 \mathrm{~V}(36.5 \mathrm{~V})$ & AC power & $0-250 \mathrm{~W}$ \\
& & DC Power & $0-190 \mathrm{~W}$ & AC frequency & $45-55 \mathrm{~Hz}$ \\
& & AC current & $0-1.14 \mathrm{~A}$ & temperature & $-40^{\circ} \mathrm{C}-+65^{\circ} \mathrm{C}$ \\
\hline
\end{tabular}

\section{Experimental software device}

Intelligent grid-connected PV system based on micro-inverter, with computer terminal connect NB-PLC communication module to allow the smartPV monitoring software for receiving signals properly. The computer installation software contains the software of monitoring and feedback control. The ORACLE database was used as a medium for connecting software and to store the collected data meanwhile, to provide a direct real-time data for the control analysis. Here, smartPV monitoring software works as "a server", and the feedback control software of the new developed program works as "client". On the same computer, a virtual network port on the ORACLE database are established for both software to transfer data, convey commands, feedback control operation, write and save warning text, and more detailed description can be referred to TCP connection. SmartPV monitoring software interface schematic and the new development software interface schematic diagram are shown in Figures 3 and 4. 


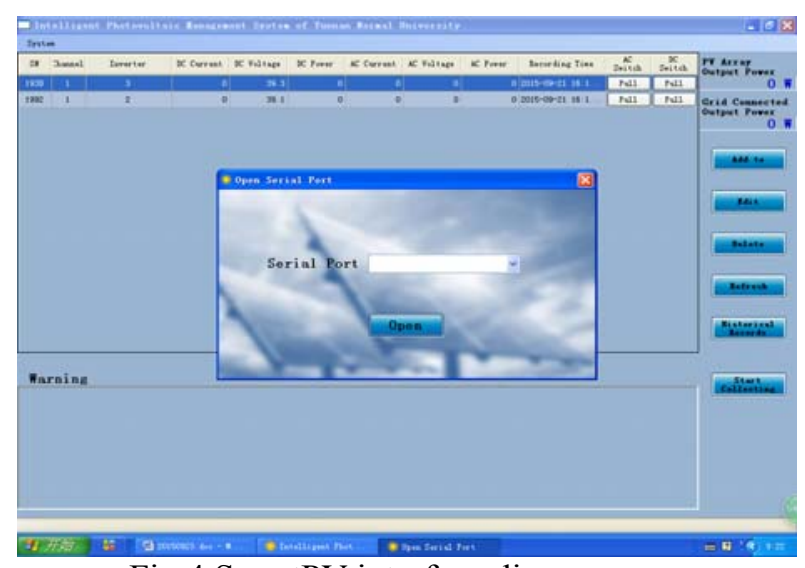

Fig.4 SmartPV interface diagram

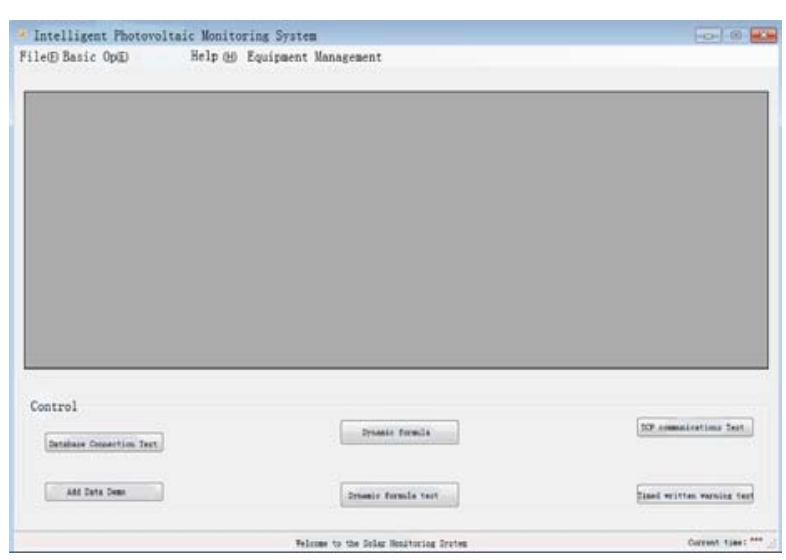

Fig. 5 The econdary development software interface

Fig. 3 shows that each solar panel and the micro-inverter are assigned channel, the smartPV acquisition software can capture the parameters of solar panels and micro inverter module level. On the detection interface, each controller can be carried out the on-off operation and historical data can be exported. The new developed software contains grouping and feedback control packet two parts, which can group and control single system for different requirements.

\section{Control principle and experimental verification}

\subsection{Control function}

For intelligent grid-connect PV system based on micro-inverters, the structure function of system acquisition and the control system for various parts diagram is shown in Fig. 6.

Fig. 6 shows that the smartPV software not only collects data into the database, but also transfers the data to history data from the database. The new developed software can be used as logic operation and fault judgment by obtaining historical data from the database, and the result of the fault will be displayed in the warning window or the relay will be operated by a request for the operation of the controller.

When the battery plate reaches the start time, the computer sends an auto-start command to the switch controller, then the system soft-stated. When the battery plate reaches the closing time, the system automatically shut down. When the battery plate is covered by persistent shadow, shadow-related information will be displayed in the warning window and saved. Consequently, by looking at the appropriate warning statement the exact time of shadow and the type of shadow area can be easily observed, so obstructions can be removed to change the impact of the irradiance of solar panels. Whenever the grid voltage becomes very high it turns off the corresponding subsystem or a single system to protect the entire PV system.

\subsection{Control process}

\subsubsection{Self-diagnose prerequisite}

The fault diagnosis method focuses on subjective shadows, panels with different shading have different output characteristics[10-16]. Therefore, three conditions were proposed to exclude the objective shadows[17] and to control the system as follows:

1. Weather: Panels power will be low in the cool days. In contrast, for sunny days the effect of using self-diagnose can be obvious and easy to find.

2. Grouping: The photovoltaic system based on micro-inverters install semicircle bracket which can rotate 360 o range in the vertical plane, and mounting bracket can be rotated 360 o horizontal range in the bottom. Therefore, for different azimuth or angle of battery plate, the power of generating can be different. If PV plates or micro-inverters are different, the power generation will be also different. Thereby, the method can ensure the comparability by classifying the different subsystems.

3. Execution time period: For different regions, seasons and sunshine are different, so the time can be also different. Power generation execution time should be selected in the daytime and on the 
peak period of sunshine.

\subsubsection{Theoretical model and model validation of shadow estimation}

This paper used the approach of special channel based on power line carrier communication technology. In order to verify the feasibility of this idea, a simulation model based on LabVIEW was built. The main control panel of the photovoltaic control is shown in Fig. 7:

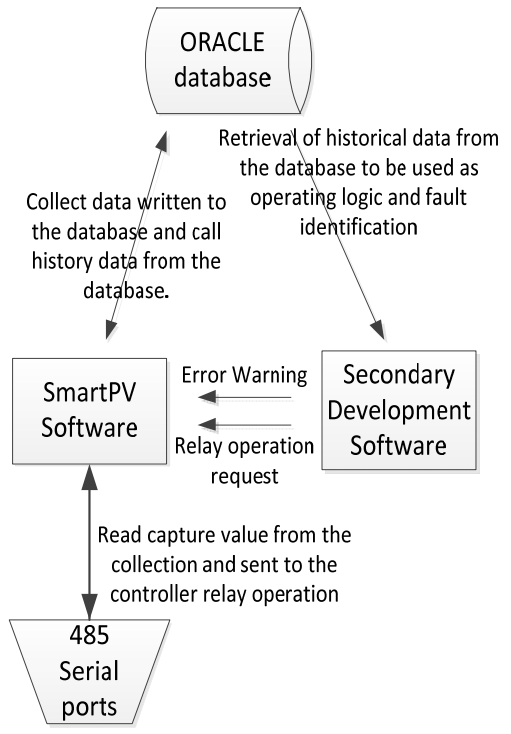

Fig. 6 functional structure of self-diagnosis method

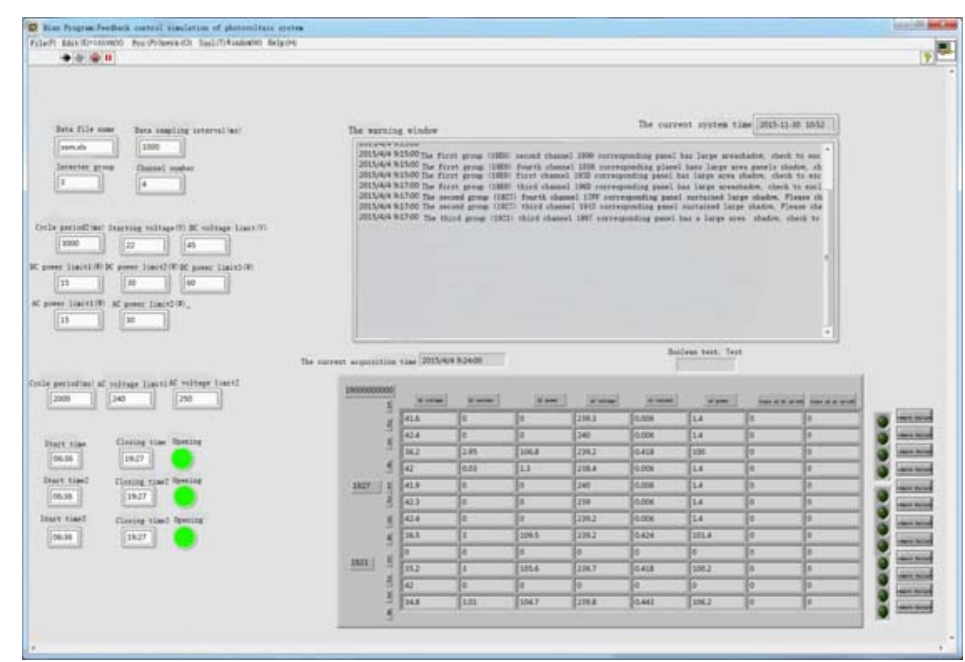

Fig. 7 Simulation panel of system self-diagnosis method

Fig. 7 shows the theory simulation model of the shadow and verifies the simulation test results

\subsubsection{Experimental flow chart}

Fig. 8 illustrates the flow chart of the experimental procedure. It was observed that after the system started it was in need to be initialized. Therefore, before processing the data, first it should be detected whether the feedback control software and ORACLE database connect success or not; then it should be detected whether self-written function is correct or not; For solar panels there was a different status, such as different orientations, different azimuths, different inverters and so on. Consequently, the whole system will be in need to different grouping and save basic management information; Then it can enter a loop feedback control of self-diagnosis: When the system reaches the start time, the system will be self-started. Subsequently, when the system covered by continuous shadow which meets the preconditions, then the shadow status will be shown in the warning window and save. When the grid voltage increased highly, the system disconnect from the grid and when the system reaches the close time, the system will be shut down; then it will be waiting for the second day to start work.

The timer can be used to judge the delay and ambiguity of the method, and it can avoid the objective shadow automatically. 


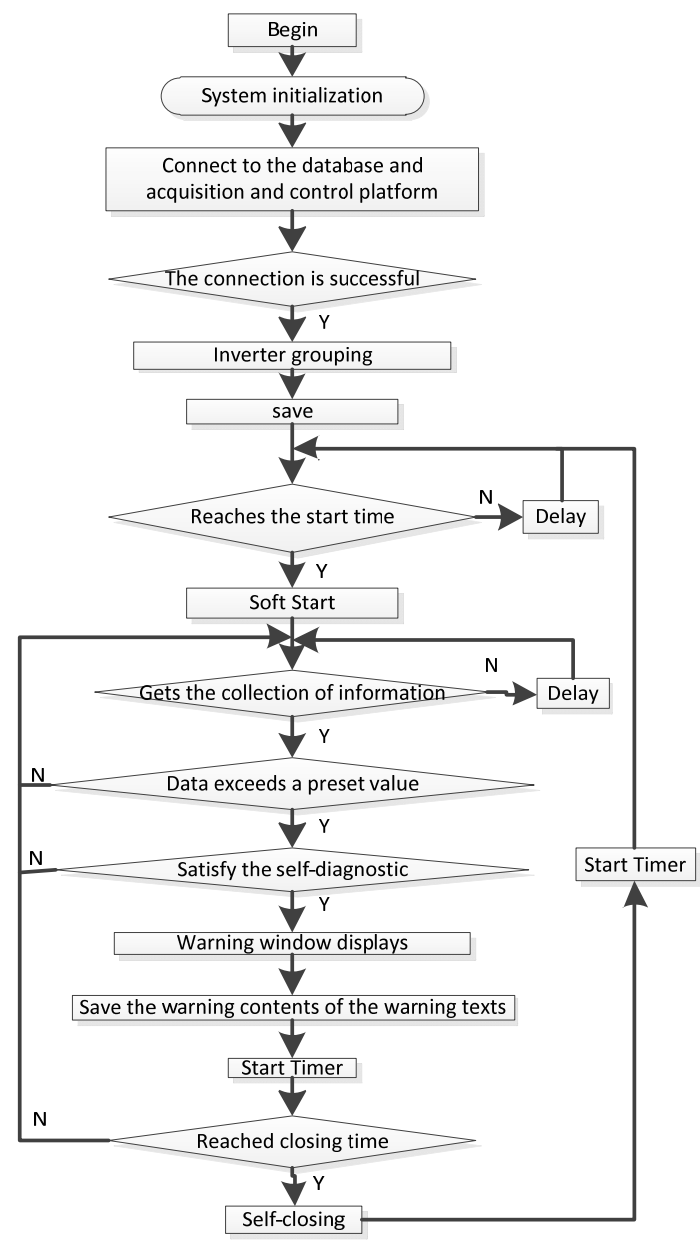

Fig. 8. self-diagnose flow chart

\subsection{Control implementation}

Combined with the existing PV information management system, the system can realize the function of intelligent photovoltaic data monitoring and online state warning management. Finally, it can achieve the group management and warning information displays of at least 4 battery boards and 4 micro-inverters. Moreover, it can realize the self-starting, self-closing. Therefore, whenever any fault occurs at the grid-connected PV system based on micro-inverters it will provide a self-close.

\subsection{Shadow self-diagnosis}

At different conditions of the battery plate shield, the feedback control management can process in real time, analyze the type of the shadow, and ultimately the shadow will be reflected in the warning window to display on the smartPV interface, and automatically write the warning statements and save the warning text under the smartPV software root directory.

In order to determine the location and the type of the shadow, the correlation information of the battery plate and the actual experiment were analyzed. The peak voltage of the battery plate is $36.5 \mathrm{~V}$, and the peak current of the battery plate is $5.21 \mathrm{~A}$. Voltage of panel with different occlusion even soared to more than $36.5 \mathrm{~V}$ or drop below $22 \mathrm{~V}$. The change of voltage amplitude was bigger and the current also changed. However, they are not as good as the power which change was large and stable. Thus, the use of reducing proportional voltage or current to define the shadow type is unreasonable. The relative proportion of power reduction was selected to determine the location and the type of the shadow. Here, the degree of shading can be divided as the following: the relative proportions of power reduction more than $15 \%$ but less than or equal to $30 \%$ is called the small area shadow; the relative proportions of power reduction more than $30 \%$ but less than or equal to $60 \%$ is 
called the general or medium shadow; the relative proportions of power reduction more than $60 \%$ is referred to as large area shadow.

When the grid voltage highly increased, it is easy to cause the damage of the micro-inverter, so the protection of the highest voltage was set at $240 \mathrm{~V}$ which the micro-inverter can withstand the highest voltage. If the grid voltage exceeds $240 \mathrm{~V}$, the feedback control system closes down the whole subsystem.

The references of variation of the output characteristic of the battery plate with shadow have been reported[18-20]. The power of the battery plate with shadow was obviously smaller than the output power of the other battery board in this group. The specific location of the shadow can be found by the relative power, then through the analysis of the voltage to find out the specific reasons. The transient state of the occlusion can be excluded by using the timer. If there is continuous shading, the warning window of the smartPV monitoring software will show the status of the shadow and save the related information for the human cleaning and maintenance. Self-diagnose feedback control interface is shown in Fig. 9.

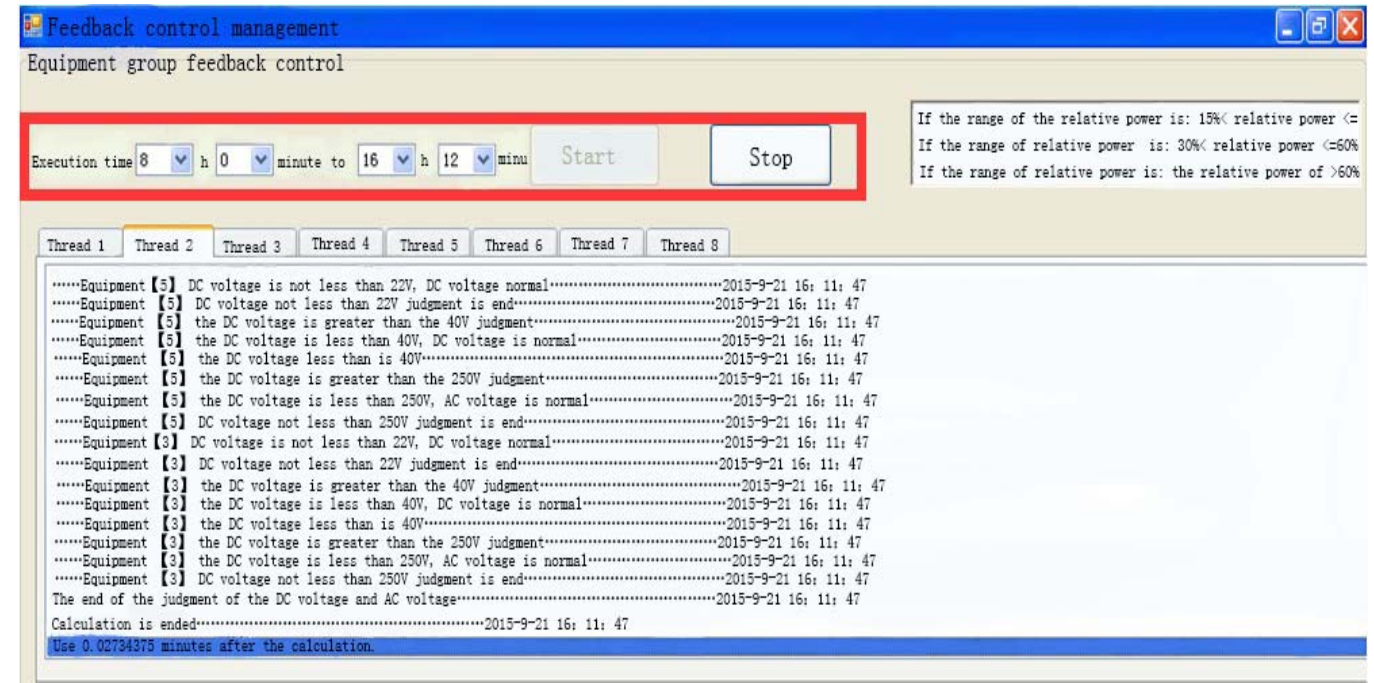

Fig. 9. Self-diagnose feedback control interface

Through the related experiments, by analyzing the acquisition data the following warning contents in warning window of smartPV monitoring software were observed and respectively the feedback control software written in sequences, as shown in Fig. 10 and Fig. 11.

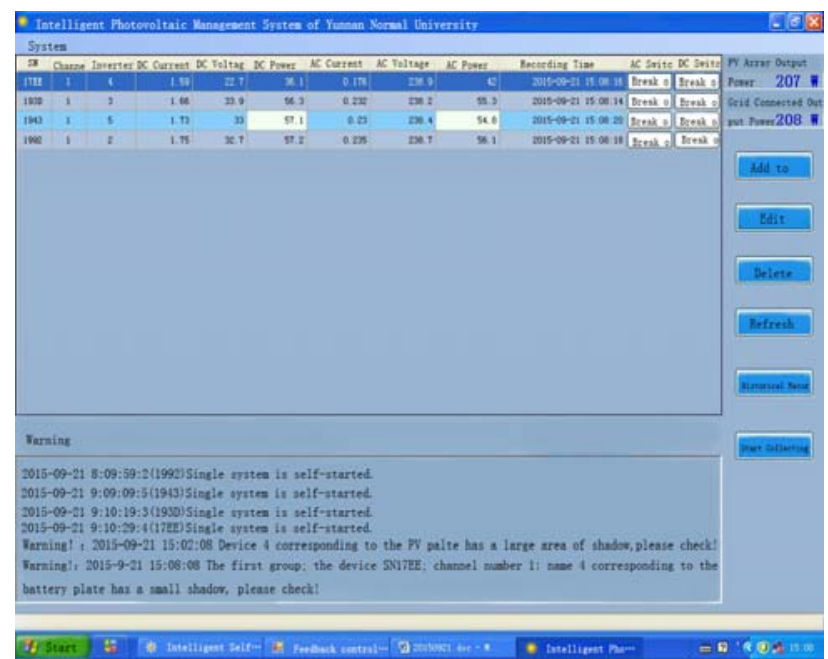

Fig. 10 warning content on smartPV interface diagram

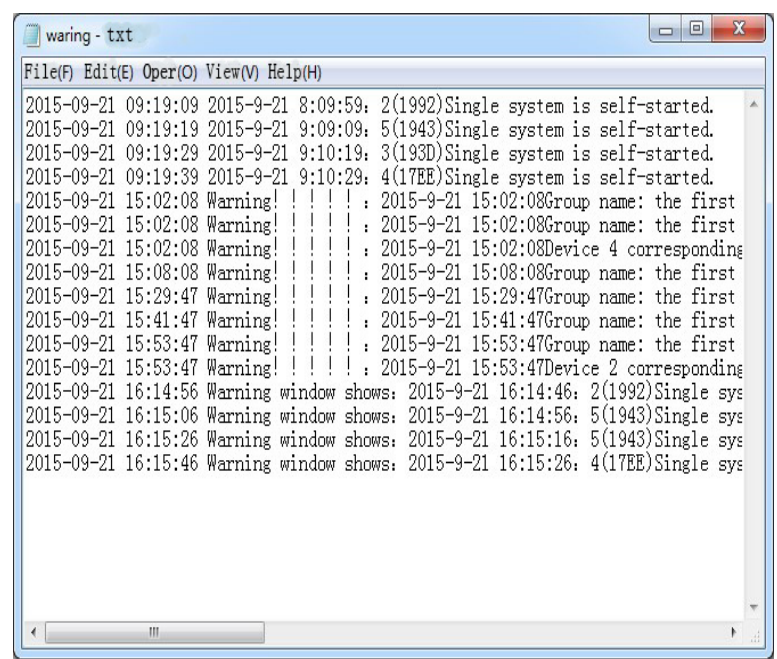

Fig. 11the warning text self-written diagram

Fig. 10 and Fig. 11 show that the experiment can achieve the purpose of feedback control self-diagnose of the online operation state detection of the smart grid-connected PV system. 


\section{Conclusion}

In this paper, a smart grid-connected PV system based on the micro-inverter, the system self-started and self-closed was controlled. Furthermore, the specific location and type of the shadow were investigated. Under reasonable conditions, the method of self-diagnose for the shadow and grid voltage was analyzed, and the on-line real-time detection was realized. The simulation model and the experimental results also confirmed the feasibility.

This method can realize the following functions: the sub-system grouping contrast, the system self-started, found the specific position of the battery plate. The degree of shading can be divided as the following: the relative proportions of power reduction more than $15 \%$ but less than or equal to $30 \%$ is called the small area shadow; the relative proportions of power reduction more than $30 \%$ but less than or equal to $60 \%$ is called the general or medium shadow; the relative proportions of power reduction more than $60 \%$ is referred to as large area shadow; protect system and the system self-closed.

The method can reduce the system costs without using light or temperature sensor or other hardware structures. The energy losses caused by the shadow were represented by the degree of shadow; the simple and accurate warning statements provide faster and more accurate information for the maintenance person. This method is simple and easy to operate, which makes the maintenance of grid-connected PV system more secure and efficient, meanwhile, it can be applied to the online state maintenance of household type and large distributed grid-connect PV system.

\section{Acknowledgement}

This research work is funded by the high efficiency and low cost thin film solar cells and smart photovoltaic systems co-research (2011DFA62380), renewable energy research and Development Cooperation Innovation Center in the southwest region (5300205020516009) and the Yunnan Province renewable energy research and innovation team (2006PY03). Thanks for the help and support of the project team.

\section{Reference}

[1]. Yuanzhang Wang, Zhihua LI, and Chunhua. Wu. A Survey of Onfine Fault Diagnosis for Photovoltaie Modules Based on Four Parameters. Proceedings ofthe CSEE, 2014. 34(13): p. 2078-2087.

[2]. Xueyu Zhang, Xueli Guo; Dayong Chang, et al.,Investigation of multilevel multifunctional grid connected inverter topologies and control strategies used in PV systems. Renewable and Sustainable Energy Reviews, 2015. 42: p. 572-574.

[3]. Mohammad Barghi Latrann and Ahmet Teke. Investigation of multilevel multifunctional grid connected inverter topologies and control strategies used in $P V$ systems. Renewable and Sustainable Energy Reviews, 2015. 42: p. 361-376.

[4]. Mohammad Monfared and Saeed Golestan. Control strategies for single-phase grid integration of small-scale renewable energy sources: A review. Renewable and Sustainable Energy Reviews, 2012. 16(7): p. 4982-4993.

[5]. Chouder A. and Silvestre. S. Automatic supervision and fault detection of PV systems based on power losses analysis. Energy Conversion and Management, 2010. 51(10): p. 1929-1937.

[6]. Yuanzhang Wang, Chunhua. Wu,Diqing Zhou, et al., A survey of fault diagnosis for PV array based on BP neural network. Power System Protection and Control, 2013. 41(16): p. 108-114.

[7]. Sotirios I. Nanou, Apostolis. G., and Stavros A. A generic model of two-stage grid-connected $P V$ systems withprimary frequency response and inertia emulation. Electric Power Systems Research, 2015. 127: p. 186-196. 
[8]. Guerrero-Rodríguez N.F., Alexis B.,Rey-Boue,Luis C, et al., Control and synchronization algorithms for a grid-connected PV system under harmonic distortions, frequency variations and unbalances. Renewable Energy, 2015. 80: p. 380-395.

[9]. Solórzano J. and Egido. M.A.. Automatic fault diagnosis in PV systems with distributed MPPT. Energy Conversion and Management, 2013. 76: p. 925-934.

[10]. Digreen Zhou; Chunhua Wu; Zhihua Li,et al. Modeling and output characteristics of PV modules under local shadow. solar energy, 2014.

[11]. Yihua Hu, Hao Chen, Ruidong Xu, et al. PV Module Characteristics Effected by Shadow Problem. Journal of Electrical Technology, 2011. 26(1).

[12]. Massi Pavan A., Mellit A., and De Pieri D. The effect of soiling on energy production for large-scale photovoltaic plants. Solar Energy, 2011. 85(5): p. 1128-1136.

[13]. Kalogirou, SA., Agathokleous R., and Panayiotou G., On-site PV characterization and the effect of soiling on their performance. Energy, 2013. 51: p. 439-446.

[14]. Lorenzo E, Moretón R, and Luque. I., Dust effects on PV array performance: in-field observations with non-uniform patterns. Progr Photovolt Res, 2014. 22: p. 666-670.

[15]. Orduz R. Analytical study and evaluation results of power optimizers for distributed power conditioning in PV arrays. Progr Photovolt Res, 2013. 21: p. 359-373.

[16]. Zorrilla-Casanova J. Losses produced by soiling in the incoming radiation to PV modules. Progr Photovolt Res, 2013. 21: p. 790-796.

[17]. Shuaijie Zhang. Shading Analysis \& Improvement for Distributed Residential Grid-Connected PVs System. Australia Solar 2014 Technology Research Forum, 2014.

[18]. Firth SK, Lomas KJ, and Rees. SJ., A simple model of PV system performance and its use in fault detection. Sol Energy, 2010. 84(4): p. 624-635.

[19]. Alonso R. Analysis of inverter-voltage influence on distributed MPPT architecture performance. IEE Trans Power Electron, 2012. 59(10): p. 3900-3907.

[20]. Femia N. Power electronics and control techniques for maximum energy harvesting in PV systems. CRC Press, 2013. 7(3): p. 66-67. 